\title{
Engineering Graduate Attributes - INVESTIGATION
}

\author{
Warren Stiver, \\ Andrea Bradford, \\ Sheng Chang, \\ Khosrow Farahbakhsh, \\ David Lubitz, \\ Joanne Ryks, \\ Bill van Heyst, \\ Hongde Zhou \\ Richard Zytner \\ School of Engineering \\ University of Guelph \\ wstiver@uoguelph.ca
}

\section{Introduction}

The Canadian Engineering Accreditation Board (CEAB) has introduced a new graduate attributes component to its overall accreditation process and assessment [1]. There are twelve graduate attributes that all programs will be required to demonstrate achievement of starting with reviews in 2014. Prior to 2014, no deficiencies will be assessed under this section.

The CEAB is not alone nor is it the first engineering accreditation body to introduce an outcomes assessment approach. The Washington Accord is an international agreement among 13 signatory countries and 4 countries with provisional status [2]. Canada is a participant in the Washington Accord that is likely a substantial driver for CEAB's introduction of graduate attributes and a primary reason the vocabulary within the CEAB attributes is quite similar to the Washington Accord.

ABET introduced outcomes assessment in 1997, following nearly a decade of development, ABET adopted Engineering Criteria 2000 (EC2000), considered at the time a revolutionary approach to accreditation criteria [3]. The earlier efforts in the U.S. offer advantages but their outcomes have greater differences.

Ontario universities also face Undergraduate Degree Level Expectations (UDLEs) that have been agreed to by Ontario Council of Academic Vice Presidents [4]. The University of Guelph also has overall bachelor degree Learning Objectives [5].

Environmental Engineering is a fully accredited engineering program in the School of Engineering at the University of Guelph. It is a blend of traditional engineering disciplines plus life and earth sciences to prepare graduates to tackle the many local to global environmental challenges the world faces.

Physical, life and earth science components of the curriculum are primarily delivered by pure science departments from across campus. Mathematics and statistics are primarily taught outside the School. Colleges of Arts, Social and Applied Human Sciences, and Management and Economics dominantly offer the complementary elective courses. The School focuses its effort on the engineering sciences and engineering design portions of the program. The School of Engineering is non-departmentalized. As a result, it has developed an engineering science core (e.g. Fluid 
Mechanics, Electric Circuits and System Analysis) and an engineering design core sequence (Engineering \& Design I to IV). Each program is then responsible for the development and delivery of its program specific engineering science and engineering design.

The School of Engineering's next accreditation visit is in the Fall of 2011. In preparation for this visit it is necessary to begin the graduate attributes work. The School didn't believe that it could tackle the entire graduate attribute domain at once. It didn't believe that it could wait for the national initiatives (the National Deans or the NSERC Chairs in Design Engineering) to reach some reasonable stage of completion. The School decided to choose one attribute as a basis to learn. It chose the INVESTIGATION attribute. Investigation attribute was chosen in part for synergy benefits with our new Engineering Complex - doubling total space through new wings and comprehensive renovations. It is a timely opportunity to reflect on the hands-on elements of the curriculum.

The CEAB's Investigation attribute is defined as:

"An ability to conduct investigations of complex problems by methods that include appropriate experiments, analysis and interpretation of data, and synthesis of information in order to reach valid conclusions." [1]

For comparison, the Washington Accord's Investigation attribute is provided as:

Conduct investigations of complex problems including design of experiments, analysis and interpretation of data, and synthesis of information to provide valid conclusions. [2]

ABET's near equivalent is criteria $3 \mathrm{~b}$ :

"an ability to design and conduct experiments, as well as to analyze and interpret data". [6]

The Environmental Engineering (EE) group embraced graduate attributes as an opportunity to enhance the EE program rather merely a task to complete.

The objective of this paper is to share with the Canadian engineering education community the EE group's initial efforts. Any engineering program in Canada could use the process undertaken. The majority of the Investigation results likely translate to most engineering programs.

\section{Investigation Attribute - what does this mean?}

The Environmental Engineering (EE) group met initially to discuss the CEAB description of the Investigation Attribute. Early questions included: "Was the description unambiguous?" or "Did it mean something different to each of us?"

In this process, the group picked at the keywords in the description and reflected on the description as a whole. The keywords identified were (in bold):

Investigation: An ability to conduct investigations of complex problems by methods that include appropriate experiments, analysis and interpretation of data, and synthesis of information in order to reach valid conclusions.

Investigation itself warrants reflection. The CEAB and Washington Accord both use Investigation within the definition of Investigation attribute leaving room for uncertainty. ABET's near equivalent criteria (3b) does not use the investigation word as the title for the attribute nor does it use investigation in the description.

Investigation has been interpreted as for the purposes of advancing learning, knowledge or understanding. At the grandest level, the advancement is research and discovery. For most undergraduate students, the investigation opens up new knowledge or understanding for the student but is otherwise well known in the field. Replacing this interpretation for the investigation word in the definition would lead to:

\footnotetext{
"Investigation: an ability to advance knowledge and understanding of complex problems through methods ...."
}

Experiments coupled with include was an important part of early discussions. The interpretation by the group was that include meant "include but not limited to" or that the word experiment should be interpreted broadly. The experiments would certainly include bench scale work but also be open to field or full scale work. The experiments or investigations would also include the domain of simulations. For Environmental Engineering, the practitioner would not be limited to narrowly defined bench scale experimentation as a means to investigate complex problems. A skilled graduate should have both capabilities, should understand the strengths and weaknesses in both and should be capable of designing an investigation that makes intelligent use of both.

The EE group also believes that a skilled graduate should be capable of intelligently dealing with data generated by others.

Complex problem was interpreted to mean the absence of an established (textbook, standard, etc.) protocol is not already available to guide the 
investigation. In Environmental Engineering, these complex problems will often be multivariable in character and be associated with serving multiple audiences.

Complexity is embedded within many attributes (problem analysis, investigation, design, engineering tools, and communication skills). Is it likely that a graduate who has demonstrated an ability to deal with complexity in one attribute is likely equipped with the capacity to deal with complexity in other attributes? Is complex design capabilities likely demonstrative of complexity skills that would then transcend to investigation? Is the reverse equally true?

Analysis of the data incorporates many elements. It frequently will include transformation of the raw data, based on established fundamentals, to derive a desire property. It can be developing new relationships. In either case, statistics plays an important role. Everything from simple averaging to multivariable regression should be part of the analysis. In complex problems, error analysis and propagation of errors is an essential element.

Interpretation relies on the ability to understand all of the steps in the investigation. Relies on understanding the limitations of the equipment and the data. Interpretation relies on the validation of the data and the analysis. Does theory agree? Does the literature agree? The ability to develop more than one interpretation of the data is seen as a particularly valuable attribute.

Synthesis is that final step associated with deriving valid conclusions from the investigation in the context and limitations of the work. In Environmental Engineering, it is believed that the ability to derive valid recommendations is of equal value and significance.

During the conversations around valid conclusions, the important connection between validity of conclusions to the appropriate definition of the problem was evident. An investigation process became an effective framework for our conversations. It is hoped that our graduates see and understand investigation as an entire process.

\section{Investigation Process}

An Investigation Process was divided into three stages. Stage 1 is Problem Definition. Stage 2 is Strategy and Execution. Stage 3 is Interpretation.

Problem definition stage is considered to include establishing the objectives of the investigation. Objectives consider the audience. An investigation linked to a legal case is different in many ways from one that it is linked to technology development. Within the problem definition stage, the EE group identified 11 elements that are a part of the process. The 11 elements are: critical thinking, single and multi-objectives, audience, recipe and open-ended, hypothesis development, single and multi-variable, and steady state and dynamic systems.

Strategy and execution involves the broad domains of planning, developing and choosing the investigation approach that will achieve the objectives AND the ability then to carry out that approach. The group identified 24 elements fitting into this part of the process. The 24 elements are: creative/novel strategies, different strategies, surrogate strategies, single and multi-step strategies, qualitative, quantitative, scale, laboratory, field, simulation, literature, standard methods, cost, environmental impacts, social impacts, risks, ethics, time, individual execution skill, expert roles, tool skills, and safe execution.

Interpretation includes data analysis, interpretation, valid conclusions and communication. Communication is an example of an interconnected attribute. It is an attribute in its own right and one that transcends all of engineering. In addition, there are communication skills specific to investigation that warrants direct attention. The group identified a total of 21 elements for the interpretation stage, they are: single and multi-variable analysis, steady state and dynamic systems analysis, linear and non-linear analysis, statistics, multiple interpretations, validation/verification, literature grounding, theory grounding, ground truthing, versus hypothesis, versus objectives, extrapolation, scale-up, recommendations, report structure, results presentation, logic argument and value reflection.

The 56 elements that have been identified are recognized as not being perfect. However, the EE group believed that it was appropriate to take a first attempt at Curriculum Mapping. The group believed that the 56 elements were sufficient to provide insights regarding our curriculum.

\section{Curriculum Mapping}

In conducting a first attempt at Curriculum Mapping a number of decisions were necessary. It was decided to limit this first map to the Environmental Engineering specific courses that are required for all Environmental Engineering students. This is a total of eight courses taken from Semester 3 to Semester 8. It was decided to survey which elements were addressed in which courses and to survey to what level. Curriculum mapping is generally seen as capable of characterizing the intentions of the curriculum.

In terms of level, it was discussed whether to assess using a measure as light as low-medium-high through 
to seemingly more sophisticated terminology. We opted to attempt to describe the level using Ideas, Connections and Extensions (ICE) framework [7]. An Idea is associated with knowledge, basic facts and essential concepts. A Connection is the ability to apply concepts and knowledge pieces in a directly introduced context. An Extension is the ability to apply ideas and connections to new contexts frequently in creative ways.

The curriculum mapping survey asked each member of the group to assess their course against the 56 elements for a "B" graduating student and to indicate what ICE level they believed a graduate should achieve by convocation day for each of the 56 elements.

\section{Results}

This first survey provided some interesting results and it is likely ideal that we recognized it as a first pass only. Only seven of the eight courses were surveyed owing to the absence of one colleague. Two of the courses benefitted from a staff and faculty view.

The convocation day ICE level to be achieved was completed by five of the group members. The results indicated some considerable spread or divergence in view. For about $25 \%$ of the elements, the spread ranged from Idea to Extension level. This divergence reflects a combination of three factors. One factor is the varied and limited comfort of the ICE framework by the participants. The ICE framework was new to the majority of the group. The group had not established examples of the different levels for each element. In hindsight, it is likely that this would have been quite beneficial and will be a valuable step as we move forward.

The second factor contributing to the range is the differences in understanding of the each of the elements. This factor was partially evident in comparison of the Interpretation stage relative to the other two stages. No Interpretation element had an Idea to Extension level spread. Whereas, $40 \%$ of the elements in the other two stages had an Idea to Extension spread in the survey response. It may be that the Interpretation stage is the most consistently understood within the group. Additional effort to gain a better, or at least more common, understanding of the first two stages would be valuable.

The final factor is just a different view regarding what is a desirable level for a graduating student. This is to be expected at this stage of our curriculum assessment. However, it is appropriate for groups or programs to reach a consensus on the targets for graduating students. Once these targets are demonstrably achieved, it is then, in the spirit of continuous improvement, appropriate to pick which elements to raise the bar.

The mapping of the individual courses against the 56 elements of the Investigation attribute does not lend itself to a simple interpretation. For all 56 elements at least one course identified a contribution. Indeed for all 56 elements at least one course identified achieving at least a Connections level.

About $20 \%$ of elements were addressed in all seven courses. Certainly it would inappropriate for $100 \%$ of elements to be addressed in all seven courses. It is unclear whether $20 \%$ is excessive. For only 4 elements, is there fewer than 4 courses contributing. This may be indicative of too much redundancy. This redundancy may be compounded when the courses outside of the Environmental Engineering set are factored in.

The progression of level through the courses is not evident through the survey. During the discussion of the survey by the group it was clear that there were inconsistencies in the level assessment by individuals. Some individuals assessed the ICE level achieved within the course relative to the graduating engineer. Other individuals assessed the ICE level in the context of the year of the course. This difference prevented a credible assessment of whether an appropriate advancement of skills was evident in the seven courses. A future pass of the survey can and should readily avoid this discontinuity.

\section{Closing, Reflection and Next Steps}

We began this process with a single graduate attribute. Some of us believed that tackling one attribute would be simple. Some of us believed that it might be quick. Some of us believed that we would do this once and be done with it. For most of us, these initial beliefs have been challenged. The process is not going to be simple, it hasn't and won't be quick and it won't be a single pass exercise. For many of us, we are recognizing that this will become the new norm. Our courses and our curriculum as a whole require ongoing and collective attention.

The process to date has raised the bar within the group in terms of understanding Investigation. Each member of the group introduced elements that broadened each of our perspectives.

During the early discussions around the Investigation attribute there was general and fairly widespread concern about the statistical element. The survey results do not reinforce this concern - the statistics element did not stand out in a negative manner. This reinforces that curriculum mapping addresses intentions only. The statistical concerns may indicate that the program is not achieving the desired 
intentions. A necessary step following mapping is to develop assessment methods. It will be interesting to see if the assessment confirms the initial concerns regarding statistics. These earlier discussions have translated into some curricular modifications.

The EE program has one course that for years has been the open-ended, experimental design course. Curriculum mapping should help the group decide the importance of embedding this experimental design dimension of this one course. These discussions and decisions are pending.

The EE group having completed this first graduate attribute step is better equipped to take the required next steps. The next steps include development of assessment and evidence building strategies, assessment of the additional attributes, a second pass at the investigation attribute for curriculum mapping, and a critical reflection of the outcome assessment work of others.

\section{References}

[1] Engineers Canada, Canadian Engineering Accreditation Board, 2009 Accreditation Criteria and Procedures, Engineers Canada, Ottawa, CAN, ISSN 1708-8054, 86p.

[2] International Engineering Alliance, Washington Accord, Washington Accord Rules and Procedures, www.washingtonaccord.org, updated 2007-08-15, 2007.

[3] ABET, www .abet.org/history.shtml, accessed May 2010.

[4] Ontario Council of Academic Vice-Presidents, Guidelines for University Undergraduate Degree Level Expecations, Dec 2005, www.cou.on.ca

[5] University of Guelph, Learning Objectives, www.uoguelph.ca/registrar/calendars/undergraduate/current/ c02/c02-learningobjectives.shtml

[6] ABET, 2009, Criteria 3b, Criteria for Accrediting Engineering Programs, www.abet.org, accessed May 2010.

[7] Young S.F. and R.J. Wilson, 2000, Assessment and Learning: The ICE Approach, Portage and Main Press. 